OPEN ACCESS

Edited by:

Li Shen,

University of Pennsylvania,

United States

Reviewed by:

Guang-Zhong Wang,

Shanghai Institute of Nutrition and

Health (CAS), China

Jie Zhang,

Fudan University, China

Shengpei Wang,

Institute of Automation (CAS), China

*Correspondence:

Jia Liu

liujiathu@tsinghua.edu.cn

Specialty section:

This article was submitted to

Artificial Intelligence in Radiology,

a section of the journal

Frontiers in Radiology

Received: 26 June 2021 Accepted: 18 November 2021

Published: 17 December 2021

Citation:

Xu S, Kong X and Liu J (2021) Expression of CRY2 Gene in the Brain

Is Related to Human Navigation.

Front. Radio. 1:731070

doi: 10.3389/fradi.2021.731070

\section{Expression of CRY2 Gene in the Brain Is Related to Human Navigation}

\author{
Shan $\mathrm{Xu}^{1}$, Xiangzhen Kong ${ }^{2}$ and Jia Liu ${ }^{3 *}$ \\ ${ }^{1}$ Faculty of Psychology, Beijing Normal University, Beijing, China, ${ }^{2}$ Department of Psychology and Behavioral Sciences, \\ Zhejiang University, Hangzhou, China, ${ }^{3}$ Department of Psychology and Tsinghua Laboratory of Brain and Intelligence, \\ Tsinghua University, Beijing, China
}

Navigation is a complex cognitive process. CRY2 gene has been proposed to play an important role in navigation behaviors in various non-human animal species. Utilizing a recently developed neuroimaging-transcriptomics approach, the present study reported a tentative link between the CRY2 gene and human navigation. Specifically, we showed a significant pattern similarity between CRY2 gene expression in the human brain and navigation-related neural activation in functional magnetic resonance imaging. To further illuminate the functionality of CRY2 in human navigation, we examined the correlation between CRY2 expression and various cognitive processes underlying navigation, and found high correlation of CRY2 expression with neural activity of multiple cognitive domains, particularly object and shape perception and spatial memory. Further analyses on the relation between the neural activity of human navigation and the expression maps of genes of two CRY2-related pathways, i.e., the magnetoreceptive and circadianrelated functions, found a trend of correlation for the CLOCK gene, a core circadian regulator gene, suggesting that CRY2 may modulate human navigation through its role in circadian rhythm. This observation was further confirmed by a behavioral study where individuals with better circadian regularity in daily life showed better sense of direction. Taken together, our study presents the first neural evidence that links CRY2 with human navigation, possibly through the modulation of circadian rhythm.

Keywords: spatial navigation, CRY2, meta-analysis, Allen Human Brain Atlas, circadian rhythm

\section{INTRODUCTION}

Navigation is of pivotal importance for survival. Previous studies have suggested that spatial navigation is highly inheritable $(1,2)$ and genetic factors play a substantial role in susceptibility to navigation-related disorders such as Alzheimer's disease (3). Recent efforts have been made to identify the genetic basis of navigation ability in human $(4,5)$, and found that the expression map of S100 calcium-binding protein B (S100B) gene in the brain is specifically correlated with functional activation for scene perception, a core component of spatial navigation (4) and that functionally unstable APOE4 gene carriers lead to a boundary-driven error correction during wayfinding (5). Because navigation is a complex cognitive process that involves multiple cognitive functions, it is likely that more genes are involved in the process. Here, we investigate whether there are other genes to construct the gene repertoire specific for navigation along with the already known navigation-related genes such as APOE4, S100B, and TOMM40 (6).

One candidate is Cryptochromes that are highly conserved across animals and associated with navigation in insects and migratory birds [e.g., $(7,8)]$. Although no direct evidence of 
Cryptochromes' involvement in human navigation has been reported, human Cryptochrome-2 (CRY2), one of the human Cryptochromes, can function as a replacement of Drosophila's inherent magnetosensor to restore the magnetic dependent navigation ability of genetically manipulated Drosophila (9), suggesting the functionality of CRY2 in navigation may be preserved in human as well. The underlying mechanism of CRY2 on navigation could involve, at least in part, two aspects. First, CRY2 might function as (a part of) the magnetosensor of a biological compass in human navigation as in non-human animals (10). In non-human animals, Cryptochromes are found to serve as a light-dependent magnetosensor [e.g., $(7,8,11)$, where the quantum spin dynamics of a radical-pair reaction in Cryptochromes change as a function of external magnetic fields and affect subsequent biochemical reactivity [e.g., $(8,11-13)]$. Although magnetosensing in human remains controversial (14, 15), one study has reported that Cryptochromes, along with human ISCA1 gene, can form magnetosensing rod-like protein complex that exhibits spontaneous alignment in magnetic fields (10). Second, human Cryptochromes are known for participating in circadian regulation $(16,17)$, and human CRY2, along with CRY1, expresses rhythmically and binds with PER proteins to inhibit the positive limb of the feedback loop including CLOCK, ARNTL/BMAL1, and ARNT2/BMAL2 (16), which activates the transcription of core clock component genes and regulates the transcription of effector clock-controlled genes to further affect various physiological functions such as cellular metabolism (18, 19). In the present study, we first explored the relation between the CRY2 gene and human navigation, and then examined the potential involvement of the two functional aspects of the CRY2 gene in navigation.

To do this, we ran an integrative study by combining gene expression data in the human brain, large-scale neuroimaging meta-analysis activation maps, and behavioral test data to examine the potential link between CRY2 and human navigation. Specifically, we examined the pattern similarity of CRY2 expression in human brain and the brain functional activation in navigation-related tasks. A similar approach that combines neuroimaging and gene expression atlas [e.g., Allen Human Brain Atlas (AHBA)] has successfully linked genes with functional activation patterns (4), functional connectivity $(20,21)$, structural connectivity (22), and alterations in brain disorders such as schizophrenia (23) and autism spectrum disorder (24). To further illustrate the functionality of CRY2 in human navigation, we examined the correlation between CRY2 expression and various cognitive components underlying navigation, such as perception, memory, and action. Finally, we examined the relation between the neural activity pattern of human navigation and expression maps of genes of two CRY2-related pathways, i.e., the magnetoreception and circadian regulation.

\section{MATERIALS AND METHODS}

\section{Datasets}

\section{Allen Human Brain Atlas}

Allen Human Brain Atlas (AHBA; http://www.brain-map.org) is a publicly available online resource for gene-expression data. The atlas characterizes gene expression [i.e., messenger RNA (mRNA) quantification] in postmortem human brain based on genomewide microarray-based measurement, for more than 20,000 genes at $~ 500$ sampling sites distributed over the whole brain [see (25) for more detail about the data collection]. Normalized expression data were used in the present study. To date (search conducted on March 30, 2017), six adult donors with no history of neuropsychiatric or neurological conditions were available in the database (ages 24, 31, 34, 49, 55, and 57 years; one female). Left hemisphere cerebral cortical data are available for all six donors, whereas right-hemisphere data are available for only two. Detailed information on donors and analysis methods is available at http://www.brain-map.org. Structural brain imaging data of each donor were used to align sampling sites into standard coordinate space.

\section{Neurosynth}

Neurosynth (http://neurosynth.org) is a platform for large-scale synthesis of task fMRI data (26). It uses text-mining techniques to detect frequently used terms as proxies for concepts of interest in the neuroimaging literature: Terms that occur at a high frequency in a given study are associated with all activation coordinates in that publication, allowing for automated term-based metaanalysis. Despite the automaticity and potentially high noise resulting from the large-scale meta-analysis, this approach has been shown to be robust and meaningful [e.g., $(4,21,26,27)]$, due to the high number of studies included.

\section{Gene, Environment, Brain, and Behavior}

Gene, Environment, Brain, and Behavior $\left(\mathrm{GEB}^{\wedge} 2\right)$ is a large-scale project aimed to investigate the associations among $\mathrm{GEB}^{\wedge} 2$. The dataset used in the present study is part of the $\mathrm{GEB}^{\wedge} 2$ project. The present study recruited 292 participants (155 females; mean age $=20.6$ years, $\mathrm{SD}=1.02$ years) from Beijing Normal University (BNU), Beijing, China. Each participant completed the San Babara sense of direction questionnaire (SBSOD) and rated one's own circadian regularity in daily life.

We operationalized navigation ability as the sum scores of SBSOD. Previous studies have shown that people have explicit and accurate knowledge of their own navigation ability (28, 29). San Babara sense of direction questionnaire is a standard questionnaire on self-reported sense of direction in a large-scale environment, and has been widely used as a reliable proxy for navigation ability $(30,31)$. It includes 16 items, such as "I am very good at giving directions" and "I very easily get lost in a new city". Participants were instructed to indicate the extent to which they agreed or disagreed with each statement on a five-point Likerttype scale. A higher total score indicates better performance in daily navigation, and thus better navigation ability.

The regularity of circadian rhythm was measured by a single question: comparing to people of similar age and educational background, do you keep regular hours in daily routine? The participants were asked to respond on a nine-point scale. The choices range from 1: very irregular to 9: very regular, a higher score indicating higher regularity.

All participants had no history of neurological or psychiatric disorders. The study was approved by the Institutional Review 
Board of BNU. Written informed consent was obtained from all participants before they took part in the experiments.

\section{Pattern Similarity Analysis of CRY2 Gene Expression in the Human Brain and Functional Activation Related to Navigation}

We firstly generated a meta-analytic map for navigation using large-scale data from Neurosynth. The term "navigation" was used, which resulted in 61 studies with 2,989 activations in total. As expected, the meta-analytic activation map covers the well-established navigation-related regions, including the hippocampus, parahippocampal gyrus, and retrosplenial cortex [e.g., $(4,32-37)]$.

Next, we extracted CRY2 gene expression in the human brain from the AHBA. Three probes, A_23_P127394, A_23_P388027, A_24_P158587, were available for this gene in the atlas. Normalized CRY2 gene expression for each sampling site and each donor was obtained. Data of the three probes were averaged at each sampling site to estimate the expression level of CRY2. This provides a vector of the normalized gene expression values in a set of sampling sites, representing the spatial distribution of CRY2 gene expression across the brain for this donor.

To obtain the spatial correspondence between navigationrelated activation and CRY2 gene expression, we first translated the location of each AHBA sampling site into MNI space with non-linear co-registration. We then performed an approximate random effect analysis implemented in alleninfo (38) to examine the pattern similarity of two maps. Briefly, we first correlated the spatial distribution of CRY2 gene expression of each donor with the statistic index of activation likelihood in navigation studies (i.e., the corresponding value in the navigation map), and reported a slope of best linear fit for each donor. We then performed a one-sample $t$-test on those estimates, to test whether the distribution of the slopes was significantly away from zero. Besides illustrating the correlation between CRY2 expression level and the probability of navigation-related activation across donors, the approximate random effect analysis allows estimating the generalizability of the observed correlation beyond the six donors.

Based on the random effect analysis, we evaluated the inter-donor repeatability of the CRY2-navigation correlation to determine whether the repeatability of a CRY2-activation correlation was statistically higher than that by chance. To do so we randomly sampled the same number of studies as in the above-mentioned navigation meta-analysis from the entire Neurosynth database, excluding those included in the navigation meta-analysis. We then generated the activation probability map for each sample and correlated this randomactivation map with the expression map of CRY2, and counted how many donors' CRY2 expression level correlated significantly with the probability of the randomly pooled neural activation (uncorrected, two-tailed). This procedure was repeated 1,000 times, generating a null distribution of the inter-donor repetition. Without any link between CRY2 and navigation, the CRY2-navigation correlation should not deviate from the null distribution, i.e. with a threshold of $\alpha=0.05$, more than $5 \%$ of random samples should have equal or higher inter-donor repeatability than the correlation in question.

In addition, given the observed link between S100B gene and brain activity related to human navigation (4), we further tested whether CRY2 contributes to navigation independently or through its association with S100B. Specifically, we extracted the expression data of S100B from AHBA. Then, we conducted a stepwise multiple regression analysis with the probability of navigation-related fMRI activation at each sampling site as the dependent variable, and the respective expression levels of CRY2 and S100B at the same brain site, as well as their interaction term as independent variables. The interaction term was calculated as the product of the standardized expression levels of CRY2 and S100B. The regression was done separately for each donor of AHBA. We then examined the significance of coefficients of each independent variable across the donors using approximate random effect analysis. We further examined the statistical significance of each of the beta coefficients with the approximate random effect analysis across donors. When one regressor was not significant for one donor, we treated its beta value as zero.

\section{Association Between CRY2 Gene Expression With Neural Activity of Cognitive Processes Involved in Navigation}

Navigation is a complex cognitive process, which involves multiple processes, for instance visual perception of key landmarks, memorizing the route, generating cognitive maps, selecting between alternative routes, etc. Next, we explored the underlying cognitive components that were modulated by the CRY2 gene.

To do so, we ran similar pattern similarity analysis between CRY2 gene expression and functional activation maps of candidate components. Here, we focused on activation maps of cognitive domains, such as perception, memory, and attention, rather than navigation itself. Note that this analysis was not intended to be a comprehensive survey, neither was it conducted in an exhaustive data-driven manner, and it was further limited by the availability of suitable key terms in Neurosynth database. Thus, in a half-heuristic-half-knowledgedriven manner, we chose nine broad domains which are supposedly involved in navigation and have been widely studied in psychology and cognitive neuroscience literature: perception (for instance, object and sense perception during navigation), memory (e.g., the retrieval of semantic and spatial memory of the to-be navigated region), social cognition (e.g., the self-referential processes and social interaction involved in navigation), higher cognitive processes (such as reasoning and decision making in choosing alternative paths), language (e.g., speech understanding and phonetic processing required to understand instruction of a navigation task), attention (e.g., spatial attention and attention shift required to attending and orienting to important landmarks), executive functions (e.g., response selection and inhibition in an environment affording multiple action possibilities), motor (such as eye movement in the inspection of the environment and motor control in order to navigate through distance), and learning (such as spatial learning 
involved in navigation in novel environment). To further break down these broad domains, three sub-domains were selected from each domain (the italic terms mentioned above were some examples, see the Results section for a full list of subdomains), with a principle to cover both the spatial and non-spatial processes (for instance, spatial memory vs. episodic memory in Memory) in each domain whenever applicable, otherwise to cover both those more and less likely to be related to navigation (for instance, scene perception vs. face perception in Perception). Some sub-domains were identified by terms consisting of two words. Some of these two-word terms were built-in feature-terms in Neurosynth, and meta-analysis can be conducted based on these terms straightaway. For the two-word terms which were not built-in features in Neurosynth, studies were extracted by searching for those labeled by both one-word terms.

The activation map for each functional domain was generated using meta-analyses in the Neurosynth. The value in each voxel of the activation map of a given domain corresponds with the likelihood of this voxel being activated in studies recognized as relevant to that domain by Neurosynth. We ran the same pattern similarity analysis as the main analysis but used the activation map of each cognitive domain.

\section{Association of Navigation-Related Activation With Genes of CRY2-Related Pathways}

Besides the exploration of cognitive components that might contribute to the CRY2-navigation link, we further investigated the functionality of CRY2 in navigation by examining the association of navigation with CRY2-related functions. Here, we focused on genes of the magnetoreception and circadianregulation pathway (see section Introduction). A full list of examined genes is presented in Table 1. The pattern similarity between gene expression and neural activation was calculated with the same method for these genes as for CRY2. The robustness of these correlations was also evaluated by comparing the results with the null distribution. In doing so, we only considered a correlation robust when it yielded six repetitions across donors (among the top 5\% in the null distribution), and those yielding five (among the top 7.4\%) repetitions a marginal trend.

\section{Behavioral Correlation Between Navigation and Circadian Rhythm}

Given the observed link between the circadian-related pathway and navigation-related brain activity, we hypothesized that circadian regularity in daily life could play a role in human navigation. To test this hypothesis, we collected measures of navigation ability and circadian regularity in a large sample of the $\mathrm{GEB}^{\wedge} 2$ project, and ran a partial correlation analysis of these two measures. Participant's sex was controlled for in the analysis.

\section{RESULTS}

We first examined the similarity between the expression map of CRY2 in the brain and the map of navigation-related
TABLE 1 | Probe names of the genes tested in the present study.

\begin{tabular}{|c|c|c|c|}
\hline Category & $\begin{array}{l}\text { Gene } \\
\text { symbol }\end{array}$ & Gene name & Probe \\
\hline Gene of interest & CRY2 & $\begin{array}{l}\text { Cryptochrome } \\
\text { circadian clock } 2\end{array}$ & $\begin{array}{l}\text { A_23_P127394 } \\
\text { A_23_P388027 } \\
\text { A_24_P158587 }\end{array}$ \\
\hline Navigation & S100B & $\begin{array}{l}\text { S100 Calcium binding } \\
\text { protein B }\end{array}$ & $\begin{array}{l}\text { A_23_P143526 } \\
\text { CUST_17042_PI4162 }\end{array}$ \\
\hline $\begin{array}{l}\text { Iron- } \\
\text { metabolism }\end{array}$ & ISCA1 & $\begin{array}{l}\text { Iron-sulfur cluster } \\
\text { assembly } 1 \text { homolog, } \\
\text { mitochondrial }\end{array}$ & $\begin{array}{l}\text { A_23_P216766 } \\
\text { A_24_P387609 } \\
\text { A_24_P75543 } \\
\text { A_32, P25253 } \\
\text { CUST_7371_Pl41626 }\end{array}$ \\
\hline \multirow[t]{10}{*}{$\begin{array}{l}\text { Core clock } \\
\text { components }\end{array}$} & CLOCK & $\begin{array}{l}\text { Clock circadian } \\
\text { regulator }\end{array}$ & $\begin{array}{l}\text { A_23_P419038 } \\
\text { CUST_16994_PI4162 } \\
\text { CUST_190_PI416408 }\end{array}$ \\
\hline & PER1 & $\begin{array}{l}\text { Period circadian } \\
\text { clock } 1\end{array}$ & $\begin{array}{l}\text { A_23_P89589 } \\
\text { CUST_12126_PI4162 }\end{array}$ \\
\hline & PER2 & $\begin{array}{l}\text { Period circadian } \\
\text { clock } 2\end{array}$ & $\begin{array}{l}\text { A_23_P209320 } \\
\text { A_23_P411162 }\end{array}$ \\
\hline & PER3 & $\begin{array}{l}\text { Period circadian } \\
\text { clock } 3\end{array}$ & $\begin{array}{l}\text { A_23_P201461 } \\
\text { A_24_P230948 } \\
\text { A_24_P291231 }\end{array}$ \\
\hline & ARNTL & $\begin{array}{l}\text { Aryl hydrocarbon } \\
\text { receptor nuclear } \\
\text { translocator-like }\end{array}$ & $\begin{array}{l}\text { A_23_P162037 } \\
\text { CUST_14423_PI4162 }\end{array}$ \\
\hline & CSNK1D & Casein kinase 1, delta & CUST_8506_PI4162 \\
\hline & CSNK1E & $\begin{array}{l}\text { Casein kinase 1, } \\
\text { epsilon }\end{array}$ & $\begin{array}{l}\text { A_23_P29263 } \\
\text { A_23_P40664 } \\
\text { A_24_P918436 }\end{array}$ \\
\hline & ARNT2 & $\begin{array}{l}\text { Aryl-hydrocarbon } \\
\text { receptor nuclear } \\
\text { translocator } 2\end{array}$ & $\begin{array}{l}\text { A_23_P83579 } \\
\text { CUST_7028_PI41626 }\end{array}$ \\
\hline & NPAS2 & $\begin{array}{l}\text { Neuronal PAS domain } \\
\text { protein } 2\end{array}$ & $\begin{array}{l}\text { A_23_P218597 } \\
\text { A_23_P415984 }\end{array}$ \\
\hline & TIMELESS & $\begin{array}{l}\text { Timeless circadian } \\
\text { clock }\end{array}$ & $\begin{array}{l}\text { A_23_P53276 } \\
\text { A_24_P231004 }\end{array}$ \\
\hline
\end{tabular}

activation (Figure 1; see section Materials and Methods). Results showed a positive correlation between these two maps, which was reproducible across all six donors of AHBA (Figure 2; Mean $_{\text {beta values }}=0.07, S D=0.03 ;$ Mean $_{r}=0.17, S D=0.06$; ps $<0.045, S D=0.018)$. Moreover, the approximate random effect analysis confirmed this correlation [Figure 2B, $t_{(5)}=$ 5.46, $p=0.003$ ], suggesting that the significant correlation could be generalized beyond the six donors. To examine the robustness of the observed correlation, we established a null distribution of inter-donor repetition of gene expressionnavigation correlation, that is, the number of donors whose CRY2 expression level significantly (uncorrected, two-tailed) correlated with the activation map of a random sample of fMRI studies. The null distribution was positively skewed $(M=1.4, S D=1.7)$. Importantly, we found that the number of repetitions among donors (six out of six) in the CRY2-navigation correlation were 

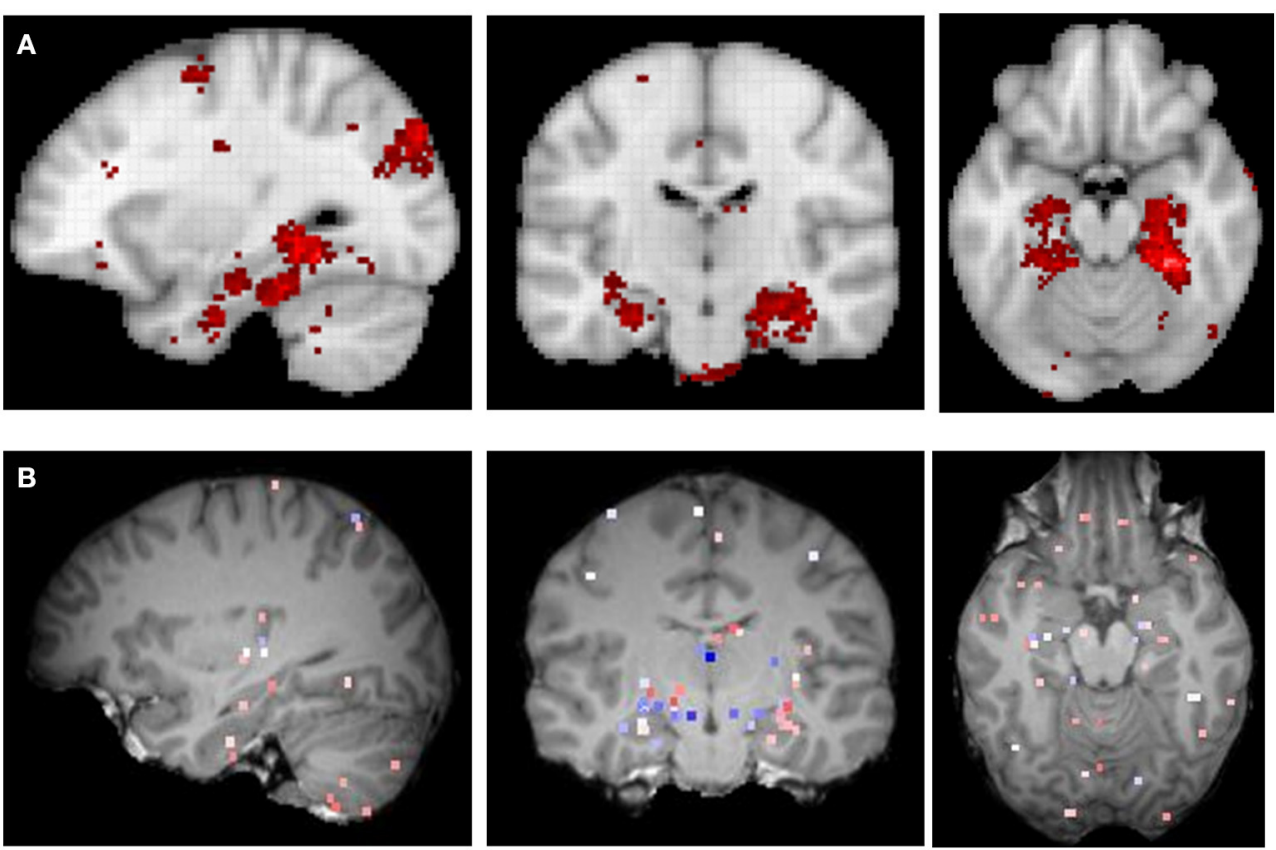

$X=-30$

$Y=-18$

$Z=-18$

FIGURE 1 | (A) The activation probability (association test) maps for navigation derived from the Neurosynth-based meta-analysis. (B) Gene expression map of CRY2 of a representative donor (donor ID: H0351.2002) from AHBA.

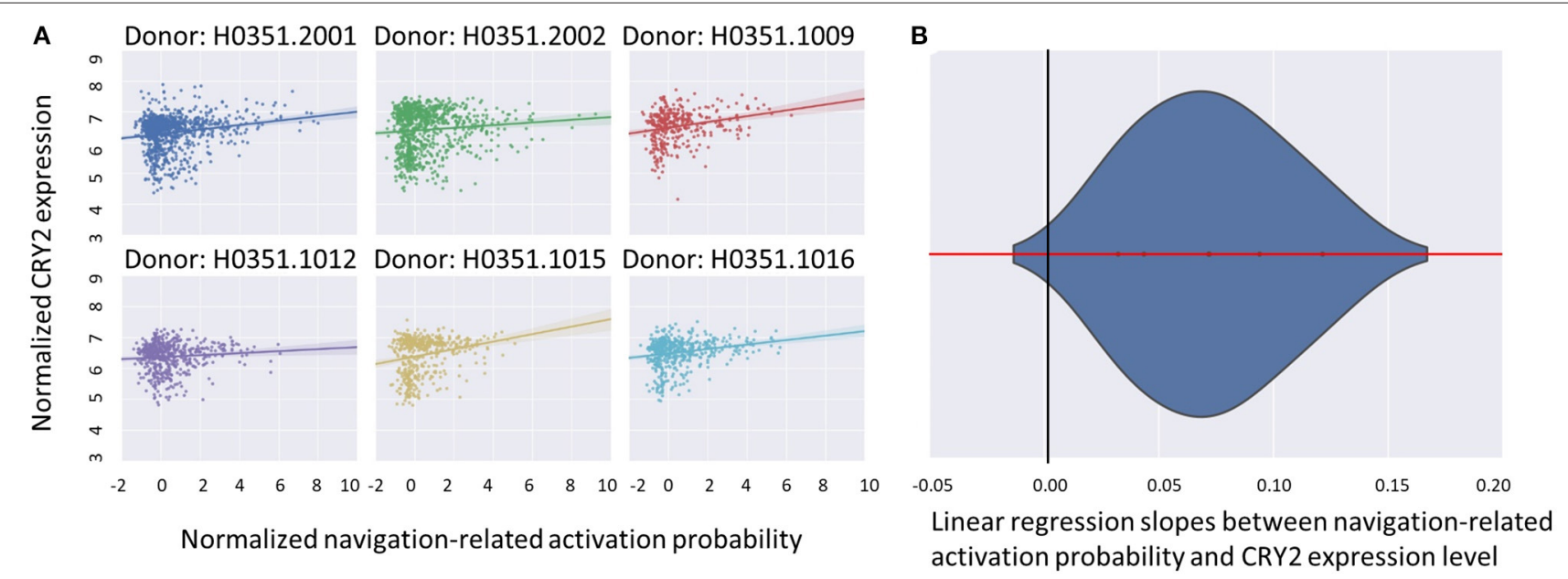

FIGURE 2 | The correlation between CRY2 expression level and the probability of navigation-related neural activities. (A) The scatter plots illustrating the correlation of each donor. The $\mathrm{X}$ axis denotes the standardized probability of navigation-related activity, while the $\mathrm{Y}$ axis denotes the standardized CRY2 expression level. Each point in the plot denotes a sample site in AHBA. The positive correlation illustrated in (A) suggested that the expression level of CRY2 is positively correlated with the probability of navigation-related activation across the brain. (B) The approximate random effect analysis estimated the distribution of the linear regression slopes between navigation-related activation probability and CRY2's local expression level, the $\mathrm{X}$ axis being the value of the slope, and the $\mathrm{Y}$ axis the probability density of a given slope value. The zero of the $X$ axis (denoted by the black vertical line) falls into the extreme left end of the distribution, suggesting the significance of the examined random effect.

among the extreme $3.1 \%$ in this distribution, which suggested that this correlation was unlikely to be caused by chance ( $p$ $=0.031$ ). In short, regions with a higher level of CRY2 gene expression were more likely to be activated in navigation-related tasks, suggesting a tentative link between the CRY2 gene and human navigation.

We also found that the expression level of CRY2 across the brain was negatively correlated with that of the navigation-related 
S100B for each donor $\left[\right.$ beta $_{\text {average }}=-0.34, t_{(5)}=7.31, p=0.001$, Supplementary Figure 1]. To rule out the possibility that CRY2 contributed to navigation through its association with S100B, we conducted a step-wise regression analysis (see section Materials and Methods), and found that, for all the donors, the resultant models were significant (Mean $R_{\text {adjusted }}^{2}=0.059, S D=0.025, p s$ $<0.02$ ). Specifically, the standardized expression level of CRY2 (Mean $\left.{ }_{\text {standardized beta }}=0.11, S D=0.10\right)$ entered the resultant models for four out of six donors ( $p s<0.045)$, the standardized expression level of S100B (Mean standardized beta $=-0.16, S D$ $=0.09$ ) was accepted for five out of six donors ( $p s<0.002$ ), while the interaction term was significant for two out of six donors ( $p s<0.009$ ). Pulling together the beta coefficients of all donors, the approximate random effect analysis revealed that the beta coefficient of expression levels of CRY2 was significantly larger than $0\left[t_{(5)}=2.87, p=0.035\right]$ and those of S100B smaller than $0\left[t_{(5)}=-4.30, p=0.008\right]$. In contrast, the beta coefficients of CRY2-S100B interaction term was not significantly different from $0(p=0.175)$. These results together suggested that CRY2 and S100B independently contributed to navigationrelated activation.

To further explore which component of navigation is associated with CRY2, we examined nine domains (i.e., perception, memory, social cognition, intelligence, language, attention, executive functions, motor, and learning) that may be relevant to navigation. Specifically, we examined the correlation between CRY2's expression map with the fMRI activation map of each of these domains extracted from the Neurosynth database (see section Materials and Methods). The results were presented in Figure 3. In general, we found that the expression level of CRY2 correlated with neural activity in multiple cognitive domains. For instance, correlations in the Memory domain generally yielded high and robust correlations, while the correlation in the domains of Perception and Social cognition

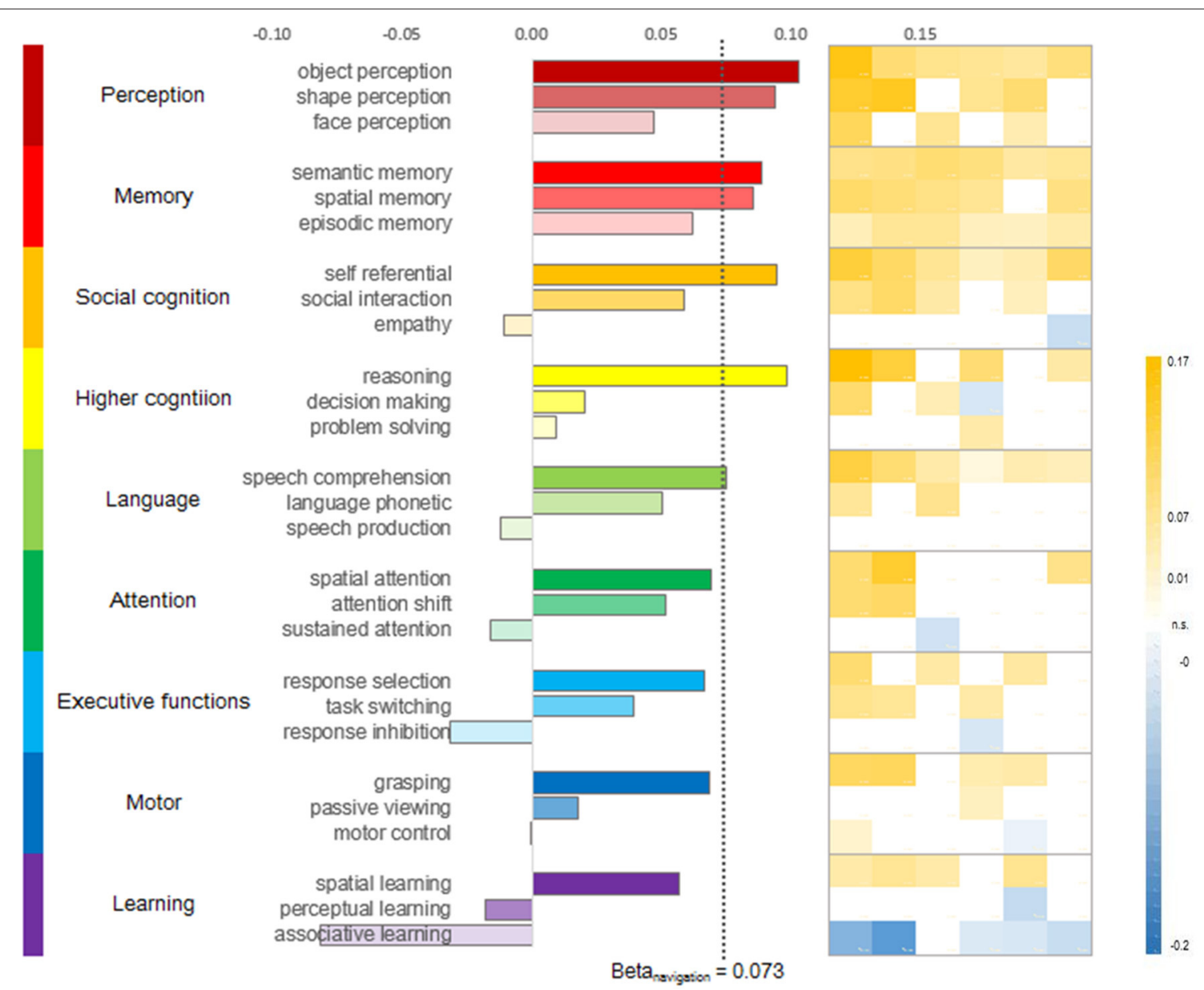

FIGURE 3 | CRY2 expression-activation correlation in various cognitive domains. Sub-domains of perception and memory strongly correlated with CRY2. In contrast, the domain of higher-level cognition and social cognition displayed variation in terms of CRY2-activation correlation. The domains of Learning, attention, reasoning and response control did not show similar correlation with CRY2. (Left) Averaged beta for the correlation between CRY2 expression level and the activation probability of respective function domain (three representative sub-domains presented). The dashed line denotes the beta value of the CRY2-navigation correlation. (Right) The beta matrix of CRY2-activation correlation across donors. Each column shows the beta values of one donor, and each row shows the beta values of the correlations between each donor's CRY2 expression level and the activation probability of the function domain denoted by the corresponding bar on the left across the brain. 
was restricted to certain sub-domains (e.g., object and shape perception in perception, and self-reference in social cognition). In contrast, in the domains of Motor and Executive function, the correlations were generally low. In addition, there was withindomain variation. For instance, in the domain of Intelligence, reasoning yielded high and robust correlation, while decisionmaking and problem-solving were not robustly correlated with CRY2. In short, instead of being associated with a specific aspect of navigation, CRY2 seemed to contribute to navigation through multiple pathways. This possibility is particularly interesting since previous studies have shown that CRY2 is involved in multiple functions, such as magnetoreception and circadianrelated functions. Next, we examined the relation between navigation and different functions of CRY2.

As for the circadian aspect of CRY2, we focused on a set of circadian regulation genes (i.e., core clock component genes), including CLOCK, CRY1, ARNTL, PER1, PER2, PER3, CSNK1D, CSNK1E, NPAS2, TIMELESS, and ARNT2 (for details, see Table 1 for the list of full names of genes and probes used in examining the expression levels). Four genes showed significant correlation (Figure 4): CLOCK, averaged beta $=0.038, p=$ 0.006 , repeated in five out of six donors; PER2, averaged beta $=0.057, p=0.045$, repeated in four out of six donors; NPAS2: averaged beta $=0.072, p=0.013$, repeated in four out of six donors; TIMELESS: averaged beta $=-0.054, p=0.030$, repeated in five out of six donors. The navigation-expression correlation was not significant for the rest core clock component genes (CRY1, ARNTL, PER1, PER3, CSNK1D, CSNK1E, and ARNT2) $\left(p_{\text {uncorrected }} \mathrm{S}>0.10\right)$. Among the genes that showed significant correlation, the CLOCK gene survived FDR correction for the multiple comparison correction $(q=0.05)$.

As for the magnetoreception aspect of CRY2, we focused on ISCA1 gene that has been proposed to form magnetoreceptor complex with CRY2. The expression map of this gene showed no significant correlation with the activation map of navigation (Figure 5).

In short, it seems that the circadian aspect, not the magnetoreception aspect, of CRY2 was related to human navigation ability. Based on this observation, we predicted that individuals with better circadian regularity likely possess better navigation ability. Indeed, college students' self-reported circadian regularity was positively correlated with self-reported sense of direction (indexed by the SBSOD score), a core component of navigation, after controlling for sex $(r=0.15$, $p=0.009$; Figure 6).

\section{DISCUSSION}

In this study, we established a tentative link between the CRY2 gene and human navigation. First, we found a significant similarity between the CRY2 expression map and fMRI activation map of navigation in the human brain. Further analyses revealed that the CRY2 gene modulated navigation possibly through various cognitive components of navigation, such as object perception and spatial memory. Moreover, instead of the speculated magnetoreception aspect, the link between CRY2 and navigation was likely based on the circadian-related function

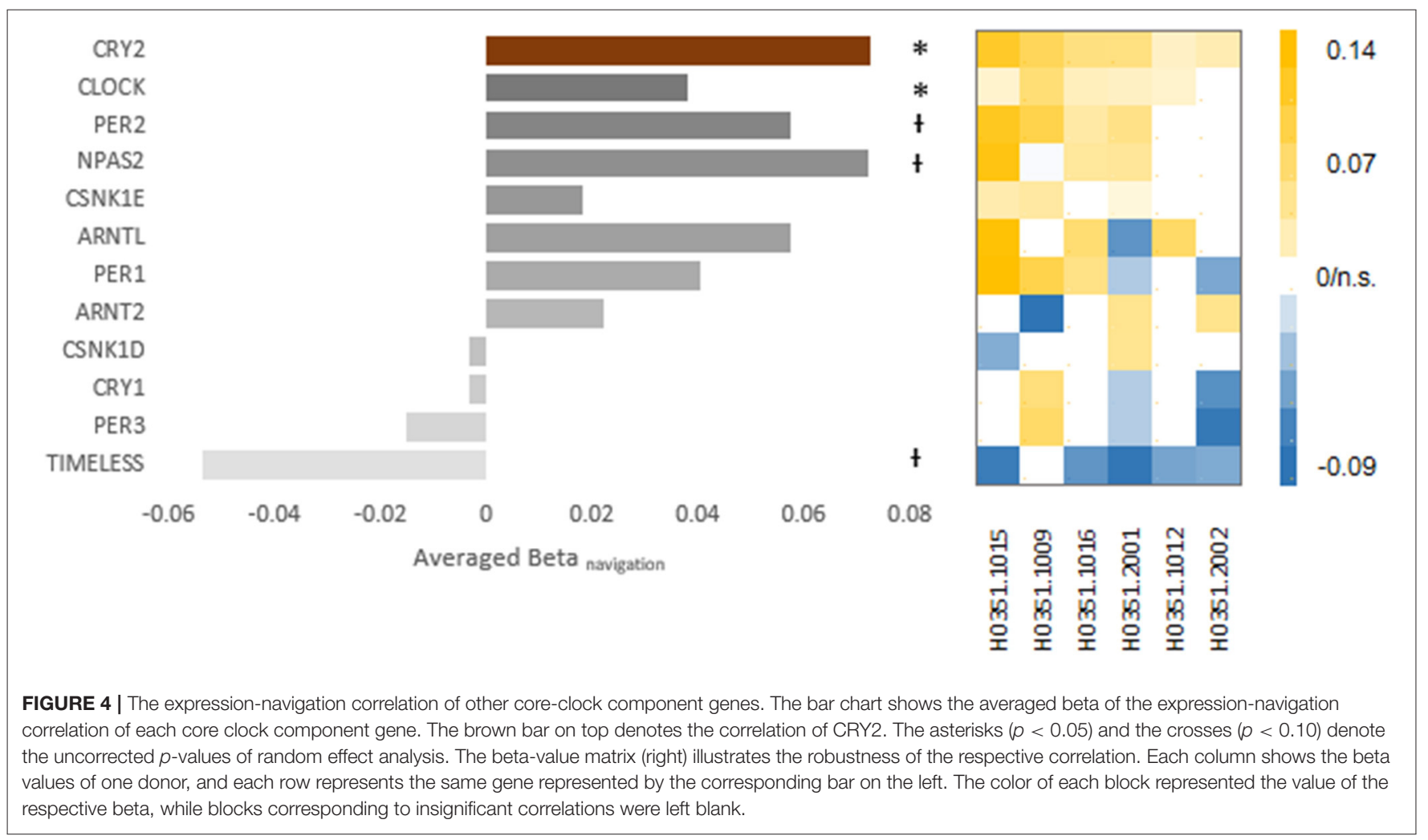




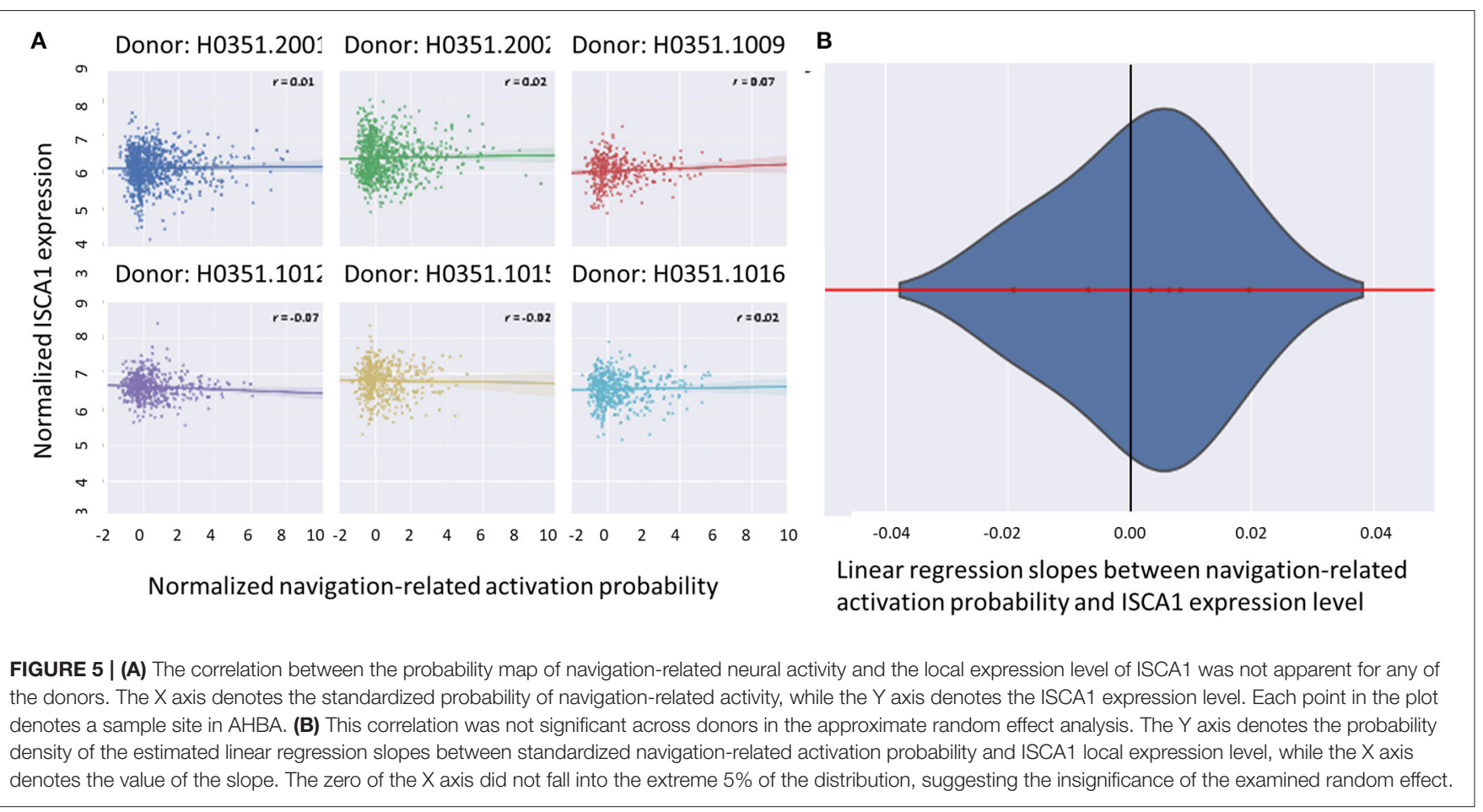

of CRY2, as the pattern similarity between human navigation and the CLOCK gene, a core circadian regulator gene, was observed. A follow-up behavioral study confirmed this finding by showing that individuals with better circadian regularity tended to have better sense of direction in daily life. In sum, our study provides the first empirical evidence linking the CRY2 gene to human navigation.

The present study demonstrated that CRY2's expression was spatially correspondent to navigation-related neural activity. However, since navigation is a complex cognitive process and involves various cognitive components, we then investigated through which of these cognitive components CRY2 affects navigation. To do this, we conducted an exploratory analysis to examine the relation between CRY2 expression and several major cognitive domains presumably related to human navigation to varying extents. We found that CRY2 correlated with multiple cognitive domains, suggesting that CRY2 might be functionally related to multiple components of navigation. Further, CRY2's expression level correlated with functional activation in different domains with different degrees of robustness as well as withindomain variation, and such variation is consistent with the presumable involvement of specific domain or subdomains in typical navigation tasks. For instance, in the memory domain CRY2 yielded robust correlations in multiple sub-domains (e.g., spatial memory and semantic memory), presumably due to the close functional relevance between navigation and memory. In contrast, the correlations in perception and social cognition were restricted to subdomains presumably relevant to navigation. For instance, in the domain of Perception, object perception that is related to scene and landmark perception in navigation yielded robust correlation; in contrast, face perception was not robustly correlated with CRY2 expression. In addition, self-referential in the social cognition domain had robust correlation with CRY2, probably due to the involvement of egocentric navigation (39-41); however, the same correlation did not exist for empathy, which is also theoretically less relevant to navigation. Taken together, the analysis on related cognitive domains suggested CRY2's association with navigation is functionally specific. Note that CRY2 has been found peak regulated in the perinatal brain (42). This suggests that the cortical development during perinatal period might be critical for navigation-related behaviors which only become observable in later developmental stages. Future studies might benefit from integrating the developmental perspective with analyses in the spatial domain such as those in the present study.

Our study further demonstrated that the association between the CRY2 gene and navigation might be realized through circadian-regulation, not magnetosensing-related, function of CRY2. Previous studies have shown that circadian regulation is a well-established function of human Cryptochromes $(16,17)$, and human CRY2 is a negative regulator in the transcriptiontranslation negative feedback loop in the molecular clock (43-45), which presents widely in mammalian tissues (45-47). Indeed, CRY2 affects various physiological functions regulated by the circadian system [e.g., (48-50)]. In our study, we found that four out of eleven of the core clock component genes, especially the CLOCK gene, showed the trend of correlation with navigation-related neural activities in the brain, suggesting a link between navigation-related neural activities and the transcriptional-translational feedback loop of circadian regulation. This link was further confirmed by the behavioral correlation between navigation ability and circadian regulation. 


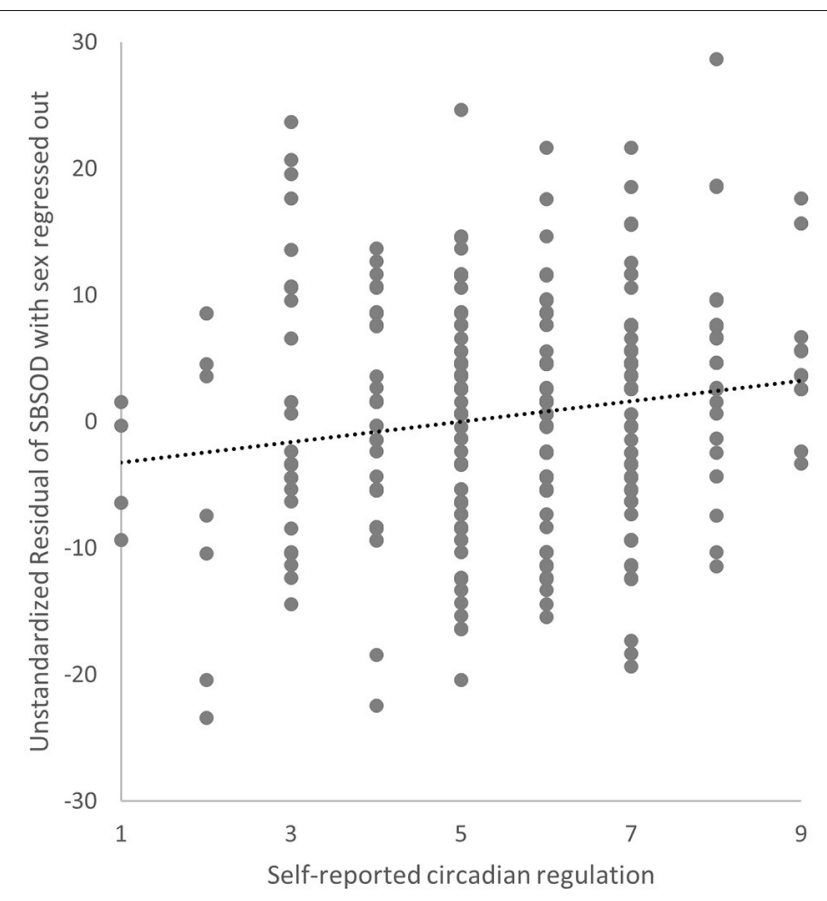

FIGURE 6 | A positive correlation between self-reported circadian regularity and navigation ability measured by SBSOD with sex regressed out. Each dot represents one individual. $X$ axis denotes the score of self-reported circadian regulation, and $\mathrm{Y}$ axis denotes the score of sense of direction after regressing out sex.

However, note that CRY2 showed higher correlation with navigation-related neural activity than CLOCK. We speculate that this difference is the result of the functional specificity of CRY2 among circadian regulation genes. For instance, the regulation of glucose homeostasis and immune function has been found relatively specific to Cryptochromes, but not apparent for other clock genes, and the relatively high expression level of CRY2 in brain regions involved in navigation might be a result of the high demand of metabolic regulation in these cortical regions, given the substantial cognitive demands in complex navigation tasks. In other words, instead of navigation ability being a readout of the entire transcriptional-translational feedback loop of circadian regulation, CRY2 might affect certain biochemical pathway in neurons involved in navigation via its rhythmic expression and its unique biochemical properties, and the CLOCK-navigation correlation might be a result of the direct transcriptional modulation between CLOCK and CRY2 $(43,44,47)$.

In contrast to circadian regulation, we did not find evidence for the CRY2 gene affecting human navigation via magnetosensing. For non-human animals, Cryptochrome's role as a light-dependent magnetosensor has been well-investigated [e.g., $(7,8,11)]$ and influential models were proposed based on the quantum spin dynamics of a radical-pair reaction in Cryptochromes change in response to external magnetic field [e.g., $(8,11-13)]$. However, magnetosensing in human has been controversial $(14,15)$. Intriguing results have been reported suggesting that human CRY2, despite having not been demonstrated in vivo, possesses the biochemical properties necessary for sensing magnetic field, and might still have the functional potential for this purpose $(9,10)$. Our study, however, did not support the conjecture that CRY2 affects navigation via magnetosensing, as we failed to find the link between the ISCA1 gene, which proposedly forms the magnetosensing complex with CRY2 as potential human biocompass (10), and navigationrelated neural activity in the human brain. Note that the present study only examined the expression of the ISCA gene in the brain because of the availability of the data, and future studies with more comprehensive data are needed to examine the relation between the magnetosensing function of CRY2 and navigation in other tissues, such as retina, where the photo-dependent cryptochrome-involved magnetoreception is likely to take place.

Combining gene expression data in the human brain, large-scale neuroimaging meta-analysis activation maps, and behavioral test data, the present study showcased how a cognitive imaging genetics approach can be used to explore the genetic basis of cognitive functions. However, several limitations shall be noted, which shall be addressed in future studies. First, our study is mainly based on correlational analyses, and the exact biochemical pathway underlying such correlation is unclear, and more genetic evidence is needed to support the present interpretations. Therefore, the cognitive imaging genetics approach used in this study can only serve as an exploratory tool, which provides clues for future fine-tuned studies. Second, in this study we relied on a meta-analysis approach to obtain brain activation map for navigation as well as for other cognitive domains. This approach, on one hand, provides a quick-and-dirty way of extensively exploring multiple cognitive functions with reasonable reliability; the results from metaanalysis, on the other hand, is unlikely to fully capture the fine structure of cognitive functions because the representativeness of available literature and the deviation between conceptual and operational definitions of cognitive functions is in question. Therefore, the finding from our study is tentative, which provides testable hypotheses for more focused and more hypothesisdriven studies.

\section{DATA AVAILABILITY STATEMENT}

The data analyzed in this study is subject to the following licenses/restrictions: $\mathrm{The}^{\mathrm{GEB}^{\wedge} 2}$ dataset used in this study are available on request to the corresponding author. Requests to access these datasets should be directed to Jia Liu, liujiathu@tsinghua.edu.cn.

\section{ETHICS STATEMENT}

The studies involving human participants were reviewed and approved by the Institutional Review Board of Beijing Normal University. The patients/participants provided their written informed consent to participate in this study. 


\section{AUTHOR CONTRIBUTIONS}

SX and JL contributed to conception and design of the study. SX performed the statistical analysis and wrote the first draft of the manuscript. All authors contributed to manuscript revision, read, and approved the submitted version.

\section{FUNDING}

This study was funded by the National Natural Science Foundation of China (31861143039 and 31600925), the

\section{REFERENCES}

1. Rimfeld K, Shakeshaft NG, Malanchini M, Rodic M, Selzam S, Schofield $\mathrm{K}$, et al. Phenotypic and genetic evidence for a unifactorial structure of spatial abilities. Proc Nat Acad Sci USA. (2017) 114:2777-82. doi: 10.1073/pnas.1607883114

2. Malanchini M, Rimfeld K, Shakeshaft NG, McMillan A, Schofield KL, Rodic $\mathrm{M}$, et al. Evidence for a unitary structure of spatial cognition beyond general intelligence. NPJ Sci Learn. (2020) 5:9. doi: 10.1038/s41539-020-0067-8

3. Jansen IE, Savage JE, Watanabe K, Bryois J, Williams DM, Steinberg $\mathrm{S}$, et al. Genome-wide meta-analysis identifies new loci and functional pathways influencing Alzheimer's disease risk. Nat Genet. (2019) 51:404-13. doi: 10.1038/s41588-018-0311-9

4. Kong XZ, Song Y, Zhen Z, Liu J. Genetic variation in S100B modulates neural processing of visual scenes in Han Chinese. Cereb Cortex. (2016) 27:1326-36. doi: 10.1093/cercor/bhv322

5. Coughlan G, Coutrot A, Khondoker M, Minihane A-M, Spiers H, Hornberger M. Toward personalized cognitive diagnostics of at-geneticrisk Alzheimer's disease. Proc Nat Acad Sci USA. (2019) 116:9285-92. doi: 10.1073/pnas.1901600116

6. Laczó J, Andel R, Vyhnalek M, Matoska V, Kaplan V, Nedelska $\mathrm{Z}$, et al. The effect of TOMM40 on spatial navigation in amnestic mild cognitive impairment. Neurobiol Aging. (2015) 36:2024-33. doi: 10.1016/j.neurobiolaging.2015.03.004

7. Zhu, H., Sauman, I., Yuan, Q., Casselman, A., Emery-Le, M., Emery, P., et al. (2008). Cryptochromes define a novel circadian clock mechanism in monarch butterflies that may underlie sun compass navigation. PLoS Biol. 6:e4. doi: 10.1371/journal.pbio.0060004

8. Rodgers CT, Hore PJ. Chemical magnetoreception in birds: the radical pair mechanism. Proc Nat Acad Sci USA. (2009) 106:353-60. doi: 10.1073/pnas.0711968106

9. Foley LE, Gegear RJ, Reppert SM. Human cryptochrome exhibits light-dependent magnetosensitivity. Nat Commun. (2011) 2:356. doi: 10.1038/ncomms1364

10. Qin S, Yin H, Yang C, Dou Y, Liu Z, Zhang P, et al. A magnetic protein biocompass. Nat Mater. (2015) 15:217-26. doi: 10.1038/nmat4484

11. Mouritsen H, Ritz T. Magnetoreception and its use in bird navigation. Curr Opin Neurobiol. (2005) 15:406-14. doi: 10.1016/j.conb.2005.06.003

12. Schulten K, Weller A. Exploring fast electron transfer processes by magnetic fields. Biophys J. (1978) 24:295-305. doi: 10.1016/S0006-3495(78)85378-8

13. Maeda K, Henbest KB, Cintolesi F, Kuprov I, Rodgers CT, Liddell PA, et al. Chemical compass model of avian magnetoreception. Nature. (2008) 453:38790. doi: $10.1038 /$ nature 06834

14. Phillips JB, Muheim R, Jorge PE. A behavioral perspective on the biophysics of the light-dependent magnetic compass: a link between directional and spatial perception? J Exp Biol. (2010) 213:3247-55. doi: 10.1242/jeb.020792

15. Close, J. (2012). Are stress responses to geomagnetic storms mediated by the cryptochrome compass system? Proc R Soc Lond B Biol Sci. 279:rspb20120324. doi: 10.1098/rspb.2012.0324

16. Reppert SM, Weaver DR. Molecular analysis of mammalian circadian rhythms. Аnnu Rev Physiol. (2001) 63:647-76. doi: 10.1146/annurev.physiol. 63.1.647
Fundamental Research Funds for the Central Universities (2019NTSS27 and 2021XZZX006), Shenzhen Institute of Artificial Intelligence and Robotics for Society (AC01202005022), and the National Basic Research Program of China (2018YFC0810602).

\section{SUPPLEMENTARY MATERIAL}

The Supplementary Material for this article can be found online at: https://www.frontiersin.org/articles/10.3389/fradi. 2021.731070/full\#supplementary-material
17. Hastings MH, Reddy AB, Maywood ES. A clockwork web: circadian timing in brain and periphery, in health and disease. Nat Rev Neurosci. (2003) 4:649-61. doi: $10.1038 /$ nrn 1177

18. Harley CW, Farrell RC, Rusak B. Daily variation in the distribution of glycogen phosphorylase in the suprachiasmatic nucleus of Syrian hamsters. J Comp Neurol. (2001) 435:249-58. doi: 10.1002/cne.1206

19. $\mathrm{Wu} \mathrm{L}$, Reddy $\mathrm{AB}$. Rethinking the clockwork: redox cycles and nontranscriptional control of circadian rhythms. Biochem Soc Trans. (2014) 42:1-10. doi: 10.1042/BST20130169

20. Richiardi J, Altmann A, Milazzo A-C, Chang C, Chakravarty MM, Banaschewski T, et al. Correlated gene expression supports synchronous activity in brain networks. Science. (2015) 348:1241-4. doi: 10.1126/science. 1255905

21. Kong XZ, Tzourio-Mazoyer N, Joliot M, Fedorenko E, Liu J, Fisher $\mathrm{SE}$, et al. Gene expression correlates of the cortical network underlying sentence processing. Neurobiol Lang. (2020) 1:77-103. doi: 10.1162/nol_a_ 00004

22. Fulcher BD, Fornito A. A transcriptional signature of hub connectivity in the mouse connectome. Proc Nat Acad Sci USA. (2016) 113:1435-40. doi: 10.1073/pnas.1513302113

23. Romme IA, de Reus MA, Ophoff RA, Kahn RS, van den Heuvel MP. Connectome disconnectivity and cortical gene expression in patients with schizophrenia. Biol Psychiatry. (2017) 81:495-502. doi: 10.1016/j.biopsych.2016.07.012

24. Romero-Garcia R, Warrier V, Bullmore ET, Baron-Cohen S, Bethlehem RA. Synaptic and transcriptionally downregulated genes are associated with cortical thickness differences in autism. Mol Psychiatry. (2019) 24:1053-64. doi: 10.1038/s41380-018-0023-7

25. Hawrylycz MJ, Lein ES, Guillozet-Bongaarts AL, Shen EH, Ng L, Miller JA, et al. An anatomically comprehensive atlas of the adult human brain transcriptome. Nature. (2012) 489:391-9. doi: 10.1038/nature 11405

26. Yarkoni T, Poldrack RA, Nichols TE, Van Essen DC, Wager TD. Large-scale automated synthesis of human functional neuroimaging data. Nat Methods. (2011) 8:665-70. doi: 10.1038/nmeth.1635

27. Helfinstein SM, Schonberg T, Congdon E, Karlsgodt KH, Mumford JA, Sabb FW, et al. Predicting risky choices from brain activity patterns. Proc Natl Acad Sci USA. (2014) 111:2470-5. doi: 10.1073/pnas.1321728111

28. Kozlowski LT, Bryant KJ. Sense of direction, spatial orientation, and cognitive maps. J Exp Psychol Hum Percept Perform. (1977) 3:590. doi: 10.1037/0096-1523.3.4.590

29. Wolbers T, Hegarty M. What determines our navigational abilities? Trends Cogn Sci. (2010) 14:138-46. doi: 10.1016/j.tics.2010.01.001

30. Janzen G, Jansen C, Turennout MV. Memory consolidation of landmarks in good navigators. Hippocampus. (2008) 18:40-7. doi: 10.1002/hipo. 20364

31. Wegman J, Janzen G. Neural encoding of objects relevant for navigation and resting state correlations with navigational ability. J Cogn Neurosci. (2011) 23:3841-54. doi: 10.1162/jocn_a_00081

32. Epstein R, Kanwisher N. The parahippocampal place area: a cortical representation of the local visual environment. J Cogn Neurosci. (1998) 392:598-601. doi: 10.1038/33402 
33. Ohnishi T, Matsuda H, Hirakata M, Ugawa Y. Navigation ability dependent neural activation in the human brain: an fMRI study. Neurosci Res. (2006) 55:361-9. doi: 10.1016/j.neures.2006.04.009

34. Epstein R. Parahippocampal and retrosplenial contributions to human spatial navigation. Trends Cogn Sci. (2008) 12:388-96. doi: 10.1016/j.tics.2008.07.004

35. Morgan LK, MacEvoy SP, Aguirre GK, Epstein RA. Distances between realworld locations are represented in the human hippocampus. JNeurosci. (2011) 31:1238-45. doi: 10.1523/JNEUROSCI.4667-10.2011

36. Dilks DD, Julian JB, Paunov AM, Kanwisher N. The occipital place area is causally and selectively involved in scene perception. J Neurosci. (2013) 33:1331-6. doi: 10.1523/JNEUROSCI.4081-12.2013

37. Sherrill KR, Erdem UM, Ross RS, Brown TI, Hasselmo ME, Stern CE. Hippocampus and retrosplenial cortex combine path integration signals for successful navigation. J Neurosci. (2013) 33:19304-13. doi: 10.1523/JNEUROSCI.1825-13.2013

38. Gorgolewski K, Fox A, Chang L, Schäfer A, Arélin K, Burmann I, et al. Tight fitting genes: Finding relations between statistical maps and gene expression patterns. Hum Brain Mapp. (2014).

39. Easton RD, Sholl MJ. Object-array structure, frames of reference, and retrieval of spatial knowledge. J Exp Psychol Learn Mem Cogn. (1995) 21:483. doi: 10.1037/0278-7393.21.2.483

40. Wang RF, Spelke ES. Updating egocentric representations in human navigation. Cognition. (2000) 77:215-50. doi: 10.1016/S0010-0277(00)00105-0

41. Mou W, McNamara TP, Valiquette CM, Rump B. Allocentric and egocentric updating of spatial memories. J Exp Psychol Learn Mem Cogn. (2004) 30:142. doi: 10.1037/0278-7393.30.1.142

42. Wang W, Wang G-Z. Twin-peak temporal regulation during human neocortical development. Cell Discov. (2019) 5:61-61. doi: 10.1038/s41421-019-0129-3

43. Kume K, Zylka MJ, Sriram S, Shearman LP, Weaver DR, Jin X, et al. mCRY1 and mCRY2 are essential components of the negative limb of the circadian clock feedback loop. Cell. (1999) 98:193-205. doi: 10.1016/S0092-8674(00)81014-4

44. Shearman LP, Sriram S, Weaver DR, Maywood ES, Chaves I, Zheng B, et al. Interacting molecular loops in the mammalian circadian clock. Science. (2000) 288:1013-9. doi: 10.1126/science.288.5468.1013
45. Sato TK, Yamada RG, Ukai H, Baggs JE, Miraglia LJ, Kobayashi TJ, et al. Feedback repression is required for mammalian circadian clock function. Nat Genet. (2006) 38:312-9. doi: 10.1038/ng1745

46. Sancar A. Regulation of the mammalian circadian clock by cryptochrome. $J$ Biol Chem. (2004) 279:34079-82. doi: 10.1074/jbc.R400016200

47. Ko $\mathrm{CH}$, Takahashi JS. Molecular components of the mammalian circadian clock. Hum Mol Genet. (2006) 15(Suppl 2):R271-7. doi: 10.1093/hmg/ddl207

48. Wisorl JP, O'Hara BF, Terao A, Selby CP, Kilduff TS, Sancar A, et al. A role for cryptochromes in sleep regulation. BMC Neurosci. (2002) 3:20. doi: 10.1186/1471-2202-3-20

49. Gorbacheva VY, Kondratov RV, Zhang RL, Cherukuri S, Gudkov AV, Takahashi JS, et al. Circadian sensitivity to the chemotherapeutic agent cyclophosphamide depends on the functional status of the CLOCK/BMAL1 transactivation complex. Proc Natl Acad Sci USA. (2005) 102:3407-12. doi: 10.1073/pnas.0409897102

50. Cao Q, Zhao X, Bai JW, Gery S, Sun H, Lin DC, et al. Circadian clock cryptochrome proteins regulate autoimmunity. Proc Natl Acad Sci USA. (2017) 114:12548-53. doi: 10.1073/pnas.1619119114

Conflict of Interest: The authors declare that the research was conducted in the absence of any commercial or financial relationships that could be construed as a potential conflict of interest.

Publisher's Note: All claims expressed in this article are solely those of the authors and do not necessarily represent those of their affiliated organizations, or those of the publisher, the editors and the reviewers. Any product that may be evaluated in this article, or claim that may be made by its manufacturer, is not guaranteed or endorsed by the publisher.

Copyright (c) $2021 \mathrm{Xu}$, Kong and Liu. This is an open-access article distributed under the terms of the Creative Commons Attribution License (CC BY). The use, distribution or reproduction in other forums is permitted, provided the original author(s) and the copyright owner(s) are credited and that the original publication in this journal is cited, in accordance with accepted academic practice. No use, distribution or reproduction is permitted which does not comply with these terms. 